\title{
Evaluation of Dengue Fever in A Tertiary Care Children Hospital of Bangladesh
}

\author{
M R Mobarak ${ }^{1}$, R Islam ${ }^{2}$, A K M T Bhuiyan ${ }^{3}$, N Akand ${ }^{4}$, F Begum ${ }^{5}$
}

${ }^{1}$ Mohammed Reaz Mobarak Professor \& Head Epidemiology \& In-Charge of High Dependency \& Isolation Unit Dhaka Shishu (Children) Hospital

${ }^{2}$ Md. Rafiqul Islam Intensivist HDU \& Isolation Unit Dhaka Shishu (Children) Hospital

3 AKM Tajuddin Bhuiyan Resident Medical Officer

${ }^{4}$ Nabila Akand

Resident Medical Officer

${ }^{5}$ Ferdousi Begum

Registrar

\section{$3,4,5$}

HDU \& Isolation Unit Dhaka Shishu (Children) Hospital

\section{Correspondence}

Prof. Mohammed Reaz Mobarak

MSc., MS, DCH, DTM \& H

Prof. \& Head

Epidemiology and In-Charge

HDU \& Isolation Unit

Dhaka Shishu (Children) Hospital

e-mail: mobarak.dk.bd@gmail.com

\section{Abstract}

Background : Dengue is a mosquito-borne (Ades Agepti) infection which in recent years has become a major public health problem. It is now endemic in more than 100 countries in Africa, America, Eastern Mediterranean, South-East Asia and Western Pacific areas. According to WHO currently approximately 50 million cases of dengue fever occur every year.

Objective : To evaluate the current clinical picture, outcome and serology of children suffering from dengue fever admitted in a tertiary care children hospital.

Methodology : It is a hospital based prospective observational study carried out among the children having Dengue infection admitted during the period of June to December 2016 in Dhaka Shishu (Children) Hospital. After obtaining informed consent 78 suspected cases of dengue fever patients were enrolled for this study. Patient with any identified specific infection or febrile illness more than 14 days and serologically dengue negative cases were excluded from the study. A detailed history, demographic variables, clinical examination and relevant laboratory investigations were assessed. The data were collected in a predesigned structured questionnaire and were analyzedwith the help of SPSS-21.

Result : Out of 78 suspected dengue cases a total of 56 cases were serologically dengue positive. Therefore 22 serologically dengue negative cases were excluded. Most common presenting symptoms were fever $(100 \%)$ followed by myalgia $76.79 \%$, retro-orbital pain $73.21 \%$, and conjunctival hemorrhage $55.35 \%$, skin rash $55.35 \%$, with other symptoms constituting the rest. Among the serologically positive dengue cases those who presented with fever for 3 days or of lesser duration, NS1 Ag was positive in 34 cases ( 43.58\%) and those who presented with fever for 6 days or more, 14 cases $(17.95 \%)$ were dengue $\lg \mathrm{M}$ positive and 8 cases $(10.25 \%)$ were dengue $\lg \mathrm{G}$ positive with accompanying low level IgM positive; platelet count 94333/ $\mathrm{mm}^{3}( \pm 68330)$, PCV $35( \pm 3.95)$ and SGPT $210 \mathrm{U} / \mathrm{L}( \pm 80)$.Among 56 dengue positive cases $31(55.4 \%)$ were male and $25(44.6 \%)$ were female, The age range was 1 years to 18 years with mean $6.66 \pm 3.69$, predominant age group was 6 years to 12 years. Final diagnosis according to WHO classification were DF $42.68 \%$, DHF-I 32.14\%, DHF-II 16.07\%,DHF-III 8.93\%. Outcome of the disease were $89.3 \%$ discharge with advice (DA),7.1\% discharge on request(DOR), 3.6\% left against medical advice(LAMA) and no death.

Conclusion : The trend of Dengue infection in Bangladesh has been changing very rapidly. In this study our observation was incidence of dengue was more in male child, peak age group being 6 years to 12 years with most admissions during the month of September. Fever with pain (body ache, retro orbital pain), headache and maculopapular rash, positive NS1Ag and dengue IgM were the main findings found in children with dengue. Most of the dengue cases were DF and DHF.I in our study.

Key words : Dengue fever, dengue hemorrhagic fever, NS1 antigen, Dengue IgM,Dengue $\lg G$

DOI: http://dx.doi.org/10.3329/nimcj.v9i1.35928

Northern International Medical College Journal Vol. 9 No. 1 July 2017, Page 274-277

\section{Introduction}

Dengue fever is a self-limiting, systemic viral illness caused by several arthropod-borne viruses. Symptomatic dengue virus infections can present with a wide range of clinical manifestations, from mild febrile illness to a lifethreating shock syndrome. It is characterized by biphasic fever, myalgia or arthralgia, rash, leukopenia and lymphadenopathy. It may increase capillary permeability. There is hemostatic abnormality, and in severe case, a protein-losing shock syndrome (dengue shock syndrome), which is thought to have an immune pathologic basis. ${ }^{1}$

Over the past three decades, globally, there has been a dramatic increase in the frequency of Dengue fever (DF), dengue hemorrhagic fever(DHF) and dengue shock syndrome 
(DSS).Approximately 2.5 billion people around the world live in dengue endemic countries of which 1.3 billion live in 10 countries of the South-East Asia Region which are dengue endemic area. ${ }^{2}$

An estimated 50 million infections including 250000-500000 cases of dengue haemorrhagic fever and 24000 deaths per year occur across approximately 100countries.Approximately 500000 cases of DHF require hospitalization each year of whom a very large proportion are children and among them roughly $5 \%$ die. Nearly $90 \%$ of the dengue infections occur in children with a risk of dying during a second attack which is nearly 15- fold higher than that of adults. The management of dengue is essentially supportive. Without proper treatment, DHF-case-fatality rates can exceed $20 \%$. With modern intensive supportive therapy, the rate can be reduced to less than $1 \% .^{3}$

In Bangladesh, thefirst documented case of dengue- like fever occurred in 1964 popularly known as "Dacca fever" which later on serologically proved as dengue fever. ${ }^{4}$ The magnitude of dengue fever was largely unknown until it took a heavy toll in 2000 (5,555 cases and 93 deaths); 2001(2,430 cases and 44 deaths); 2002 ( 6,104 cases and 58 deaths). ${ }^{2}$

The objectives ofthis study were to know the demographic profile, clinicalfeatures, serology and outcome of dengue patients admitted at Dhaka Shishu (Children) Hospital.

Methodology: This study was a prospective observational study carried out on suspected dengue fever patients who were admitted at Dhaka Shishu (Children) Hospital, during the period of June to December 2016. Children were 1 year to 18 years of age. After obtaining informed consent from the parents, a total of 78 children suspected dengue fever (based on clinical features) were primarily enrolled for this study. Patient with any identified specific infection or febrile illness more than 14 days and serologically dengue negative cases were excluded from the study.

Fifty six (56) serologically positive (NS1 antigen, IgM, IgG antibodies) dengue patients were finally selected for our study. Dengue serology (Rapid qualitative immune-chromatographic test -NS 1 antigen was done in patients who presented with fever for 3 days or of lesser duration and Dengue IgM and IgG were done in those who came with fever for more than 5 days.

The cases were classified according to WHO Comprehensive Guidelines for Prevention and Control of Dengue and Dengue Hemorrhagic fever, 2011. The variables recorded were demographic profile, clinical manifestations, laboratory parameters (complete blood count (CBC) including white blood cell count (WBC), platelet count, PCV, SGPT and outcome of illness.

Data were collected in a predesigned structured questionnaire and recorded on MS excel sheet and were analysed with the help of SPSS 21 version.

Results : Among 78 suspected dengue cases 56 (71.8\%) cases were serologically dengue positive. Therefore 22 (28.2\%) serologically dengue negative cases were excluded from the study. Among the 56 serologically dengue positive patients 34 $(60.72 \%)$ were NS1 antigen positive, 14 (25\%) IgM antibodies and $8(14.28 \%)$ both IgM and IgG antibodies positive. The distribution of the serologically dengue positive patients out of 78 clinically suspected cases were shown in Table I.

$\begin{array}{lcc}\text { Table I: Distribution of the serologically dengue positive patients } \\ \text { Number } & \text { percentage } \\ \text { Total serology positive } & 56 & 71.8 \\ \text { Total serology negative } & 22 & 28.2 \\ \text { Serological test } & \text { Positive } & \text { percentage } \\ \text { NS1 Ag } & 34 & 60.72 \\ \text { IgM } & 14 & 25.00 \\ \text { Both IgM and IgG } & 8 & 14.28\end{array}$

Among the study patients $31(55.44 \%)$ were male and $25(44.56 \%)$ were female (fig. 1) ; 50 (89.3\%) from urban and 6 $(10.7 \%)$ rural area (fig. 2). The age of the children range from 1 year to 18 years with a mean age of $6.66 \pm 3.69$ years. The age distribution of the patients are shown in Table II.

Fig 1: Gender distribution of the patients

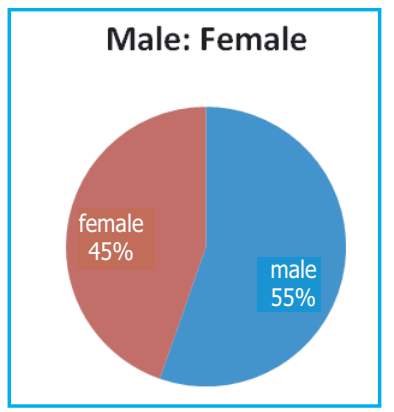

Fig 2 : Geographicaldistribution of the patient

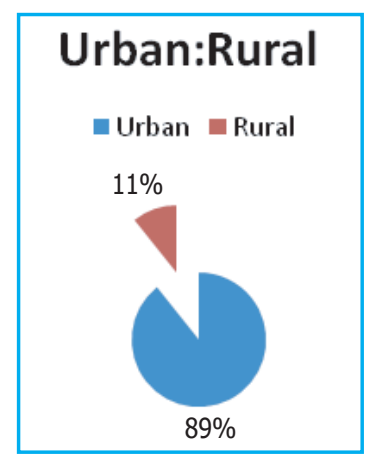


Table II : Age distribution of the patients

$\begin{array}{lcc}\text { Age range } & \text { No. }(\%) & \text { Total, No (\%) } \\ 1 \text { years-2years } & 7(12.5 \%) & \\ 3 \text { years-5years } & 14(25 \%) & \\ 6 \text { years-12 years } & 25(46.4 \%) & \\ 13 \text { years }-18 \text { years } & 10(16.1 \%) & 56(100 \%)\end{array}$

The number of the patients increased progressively from June to maximum in the month of September and then declined. The monthly distribution of the dengue patients is shown in figure 3 .

Fig 3 : Monthly distribution of the Dengue patients Admission during study period at DSH

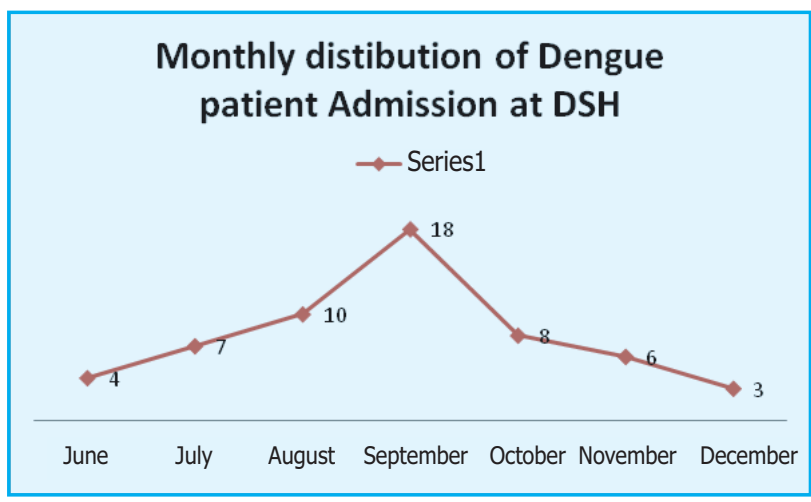

The common clinical manifestation were fever (100\%), Myalgia (76.79\%), headache $(68.58 \%)$, arthalgia $62.55 \%$, conjunctival haemorrhage $55.35 \%$, Skin rash $55.3 \%$, vomiting $51.79 \%$,and itching $51.79 \%$ and abdominal pain (46.43\%). The clinical profile of the patients were shown in table III.

Table III: clinical features of the patients of dengue fever

$\begin{array}{lcc}\text { Clinical features } & \text { No. } & \% \\ \text { Fever } & 56 & 100 \\ \text { Myalgia } & 43 & 76.79 \\ \text { Retro - orbital pain } & 41 & 73.21 \\ \text { Headache } & 38 & 68.58 \\ \text { Arthralgia } & 35 & 62.55 \\ \text { Conjunctival hemorrhage } & 31 & 55.35 \\ \text { Skin rash } & 31 & 55.35 \\ \text { Itching } & 29 & 51.79 \\ \text { Vomiting } & 29 & 51.79 \\ \text { Abdominal pain } & 26 & 46.43 \\ \text { breathlessness } & 8 & 14.29\end{array}$

On laboratory investigations the mean $\mathrm{Hb}$ was $11.19 \mathrm{gm} / \mathrm{dl}$ with SD \pm 1.29 , mean WBC was 6111 /cumm with SD \pm 2843 /cumm and mean platelet count 94333 /cumm with SD \pm 68330 . The mean PCV was 35 with SD \pm 3.95 , SGPT mean 210IU/Lwith SD \pm 84 . The laboratory parameters of the serologically positive dengue patients were shown in Table IV.
Table IV : Laboratory parameter of the patients of Dengue fever

$\begin{array}{lcc}\text { Parameter } & \text { Mean } & \text { SD } \\ \text { Hemoglobin gm/dl } & 11.19 & \pm 1.290 \\ \text { Total WBC count } & & \\ \text { per } \mathrm{mm}^{3} & 6111 & \pm 2843 \\ \text { Platelet count } & & \\ \text { per } \mathrm{mm}^{3} & 94333 & \pm 68330 \\ \text { PCV } & 35 & \pm 3.95 \\ \text { SGPT U/L } & 210 & \pm 84\end{array}$

According to WHO Comprehensive Guidelines for Prevention and Control of Dengue and Dengue Hemorrhagic fever, 2011 our study patients were classified as Dengue Fever (DF)-42.86\%, Dengue Hemorrhagic Fever-I (DHF-I)-32.14\%, Dengue Hemorrhagic Fever-II (DHF-II)-16.07\%, Dengue Hemorrhagic Fever-III (DHF- III)- 08.93\% and Dengue Hemorrhagic Fever-IV (DHF-IV) were nil (Table-V).

Table V : Distribution patients according to WHO classification

$\begin{array}{lcc}\text { WHO classification of dengue } & \text { No } & \% \\ \text { DF } & 24 & 42.86 \\ \text { DHF-I } & 18 & 32.14 \\ \text { DHF-II } & 9 & 16.07 \\ \text { DHF-III } & 5 & 08.93 \\ \text { DHF -IV } & 00 & 00 \\ \text { Total } & 56 & 100\end{array}$

Outcome of the disease were $89.3 \%$ discharge with advice (DA), $7.1 \%$ discharge on request (DOR), 3.6\% left against medical advice (LAMA) and no death (table VI).

Table VI : Outcome pattern of dengue patients

$\begin{array}{lcc}\text { Discharge pattern } & \text { No } & \% \\ \text { DA } & 44 & 78.56 \\ \text { DOR } & 7 & 12.40 \\ \text { Transfer to ICU } & 3 & 05.34 \\ \text { LAMA } & 2 & 03.70 \\ \text { Death } & 00 & 00\end{array}$

\section{Discussion}

In the present study, among 78 suspected dengue cases 56 $(71.8 \%)$ cases were serologically dengue positive. Therefore these serologically dengue positive cases were selected for our study. Among the 56 patients 34 (60.72\%) were NS1 antigen positive, 14 (25\%) IgM antibodies and $8(14.28 \%)$ both IgM and IgG antibodies positive. Isolated IgM and both IgM and IgG antibodies for dengue virus were positive in $17.95 \%$ and $10.25 \%$ of patients respectively which were much lower than that of Shahidul ABM et al's study findings (IgM and IgG antibodies for dengue positive in $40.7 \%$ and $24 \%$ cases 
respectively and both positive in $31 \%$ cases). ${ }^{5}$

In the present study, the majority of the patients (46.4\%) belong to the 1 years to 18 years age group with a male (55.44\%) preponderance. Sarker et $\mathrm{al}^{6}(2012)$ and Ahmed et al ${ }^{7}$ (2001) reported that maximum number of cases were below10 years. $^{7}$

Both Gupta et al (2006) and Chakrabarti et al (2005) also reported maximum cases of male preponderance. ${ }^{8,9}$ The majority of the patients were urban residents, this might be due to the location of the hospitals in the urban areas, additionally, urbanization also favors vector breeding.

In our study we found that the number of the dengue patients increased progressively from the month of June to maximum in the month of September and then declined. The majority of the patients were reported during monsoon and post-monsoon seasons, in accordance with the earlier reports of the dengue transmission. ${ }^{10}$

The common presenting features of the patients were fever $(100 \%)$, myalgia $(76.79 \%)$, retro-orbital pain $(73.21 \%)$, headache $(68.58 \%)$, arthralgia $(62.55 \%)$, conjunctival haemorrage $(55.35 \%)$, skin rash $(55.35 \%)$, itching $(51.79 \%)$, vomiting $(51.79 \%)$ and abdominal pain $(46.43 \%)$. These findings are quite similar to the obsevations of Shahidul ABM et al(2009) but differ from that of Ahmed et al (2001) and Rahman et al(2002). 5, 7,11 Both Ahmed et al (2001) and Rahman etal (2002) found headache was the most predominant signs, $85 \%$ and $91 \%$ respectively. ${ }^{7,11}$

In our study the rashes were $55.35 \%$ which was much lesser than that of Shahidul ABM et al (2009) and it was $75.9 \% .{ }^{11}$ In the present study the mean platelet count of the patients was $94333 / \mathrm{mm}^{3}$ which was below $1,00,000 / \mathrm{mm}^{3}$ The mean PCV was found $35 \%$ and the mean SGPT was $210 \mathrm{U} / \mathrm{L}$. These findings were all similar to the observations of Shahidul ABM et $\mathrm{al}(2009)^{5}$

There was no mortality in our study whereas it was $3.8 \%$ in Agarwal et al study (1996), 10.91\% in Wali et al study (1997), $3.8 \%$ in Rahim et al study(2006) and 6\% in ABM Shahidul ABM et al study (2009). ${ }^{5,12-14}$ In our study, DSS was a rare event, resulting in a lower case-fatality rate for dengue than reported elsewhere.

\section{Conclusion}

Inour observation the incidence of dengue was more in male child, peak age group was being 6 years to 12 years with most admissions during the month of September. Fever with pain (body ache, retro orbital pain), headache and maculopapular rash, positive $\mathrm{NS}_{1} \mathrm{Ag}$, dengue IgM were the main findings found in children with dengue. Most of the dengue cases were DF and DHF-I in our study.

\section{References}

1. Behrman, Richard E; Klieg man, Robert $M$; Halstead B. Dengue Fever. Nelson Textbook of Pediatrics, 20th edition, 2016; 1630-1632.

2. World health organization, regional Office for South-East Asia. Comprehensive guidelines for prevention and control of dengue and dengue haemorrhagic fever. Revised and expended edition. SEARO Technical Publication Series No.60 ISBN 978-92-9022-387-0@World Health organization

3. Gubler DJ The global pandemic of dengue/dengue haemorrhagic fever: current status and prospects for the future. Ann Acad Med Singapore. 1998;27:227-34 [PubMed]

4. Aziz M , Graham RR, Gregg MB. "Dacca fever" An outbreak of dengue. Pak J Med Res 1967;6:83-9

5. ABM Shahidul Alam, S Anwar Sadat. Zakaria Swapan. Clinical Profile of Dengue fever in Children. Consists of 4 serotypes (DEN 1-4). In Bangladesh. Bangladesh J Child Health 2009; VOL 33(2) : 160-164.

6. Sarker A, Tara PHDAR d , Chatterjee S. Molecular typing of dengue virus circulating in Kolkata India in 2010. Journal of tropical medicine. 2012 Feb $13 ; 2012$

7. Ahmed FU, Mahmood BC, Sharma JD, Hoque SM, Zaman R, Hasan MS. Dengur and Dengue Haemorrhagic fever in Children during the 2000 outbreak in Chittagong, Bangladesh. Dengue Bulletin 2001;25:33-39

8. Gupta E, Dar L, Kapoor G, Broor S the changing epidemiology of dengue in Delhi, India. Virology Journal. 2006 Nov 5;3(1);1.

9. Chakravarti a, Kumaria R. eco-epidemiologycal analysis of dengue infection during an out break of dengue fever, india. Virology journal.2005 Apr $14 ; 2(1): 1$

10. Anuradha S, Singh NP, Rizvi SN, Agarwal SK, Gur R, Mathur MD The 1996 outbreak of dengue hemorrhagic fever in Delhi, India. Southeast Asian J Trop Med Public Health. 1998;29:503-6

11. Rahman M, Rahman K, Siddque AK, Shoma S, Kamal AH, Ali KS, et al. First Outbreak of Dengue Hemorrhagic Fever, Bangladesh. Emerg Infect Dis 2002; 8: 738-40.

12. Agarwal R, Kapoor S, Nagor R, Misra A et al. A clinical study of the patients with DHF during the epidemic of 1996 at Lucknow, India. Southeast Asian J trop Med public Health. 1999 Dec; 30(4): 735-40.

13. Wali JP, Biswas A, Handa $R$ et al. Dengue hemorrhagic fever in adults a prospective study of 110 cases. Trop Doct. 1999 Jan; 29(1): 27-30.

14. Rahim MA, Azad MAK. Dengue: past, present and future- A review. Bangladesh J Medicine 2006; 17: 27-39. 